\title{
Restauración de sistemas de potencia usando un modelo de programación lineal entera mixta
}

\author{
Ricardo A. Pardo ${ }^{1}$ y Jesús M. López-Lezama² \\ (1) Expertos en Mercados XM S.A. E.S.P. - Filial de ISA, Calle 12 Sur No. 18-168, Medellín - Colombia. \\ (correo-e: ricardo.pardo@udea.edu.co) \\ (2) Departamento de Ingeniería Eléctrica, Facultad de Ingeniería, Universidad de Antioquia, Calle 67 No 53-108, \\ Medellín, Colombia (correo-e: jmaria.lopez@udea.edu.co).
}

Recibido Jun. 23, 2020; Aceptado Ago. 20, 2020; Versión final Sep. 15, 2020, Publicado Dic. 2020

\begin{abstract}
Resumen
El objetivo de este trabajo es proporcionar una herramienta de optimización para llevar a cabo el restablecimiento de un sistema de potencia tras un apagón a gran escala. Se propone un modelo de programación lineal entera mixta para abordar el problema de restablecimiento de sistemas de potencia. Para validar el modelo propuesto se utilizan dos sistema de prueba: 1) un sistema didáctico de 4 generadores y 2) el sistema IEEE-39 RTS. Los resultados en los dos sistemas de prueba muestran la efectividad y robustez del modelo propuesto. En particular, los resultados obtenidos con el sistema de prueba IEEE-39 RTS muestran que el modelo puede ser implementado en aplicaciones reales. Dada la naturaleza del modelo, se puede concluir que este garantiza la obtención de una solución óptima global, que indica la secuencia de unidades de generación que deben entrar en servicio, minimizando el tiempo del restablecimiento.
\end{abstract}

Palabras clave: programación lineal entera mixta; restablecimiento; sistemas de potencia

\section{Power system restoration using a mixed integer linear programming model}

\begin{abstract}
The objective of this research study is to provide an optimization tool for power system restoration after a largescale blackout. A mixed integer linear programming model is proposed to approach the restoration problem of power systems. To validate the proposed model, two testing systems are used: 1) a four generator didactic system and 2) the IEEE-39 RTS system. The results in both systems show the effectiveness and the robustness of the proposed model. In particular, the results obtained using the IEEE-39 RTS testing system show that the model can be implemented in real applications. Given the nature of the model, it can be concluded that the model guarantees the achievement of a global optimal solution, which indicates the sequence of generation units that must be set online, minimizing restoration time.
\end{abstract}




\section{INTRODUCCIÓN}

La confiabilidad de los sistemas de potencia es un aspecto que debe ser tenido en cuenta, tanto en la planeación de la operación (Quintero et al., 2019) como en el planeamiento a largo plazo (Saldarriaga-Zuluaga et al., 2018). Las sociedades modernas dependen en gran medida de la electricidad para su funcionamiento. Es por esto que después de un apagón a gran escala se debe restablecer lo más rápido posible el servicio de energía eléctrica. En este proceso se deben tener en cuenta criterios de confiabilidad y seguridad para evitar un segundo apagón (Ketabi et al., 2019). Un abordaje común en el restablecimiento es dividir el proceso en tres etapas: preparación del parque de generación, restablecimiento de la red de transmisión y atención de la demanda. El hilo conductor de estas etapas es la disponibilidad de generación; pues con una capacidad de generación disponible es posible atender la demanda del sistema eléctrico de potencia (SEP) y mantener en todo momento el balance generación-demanda necesario para estabilizar el sistema y devolverlo a su condición de operación normal.

Las plantas de generación de un SEP se clasifican en unidades de arranque en negro (BS por sus siglas en inglés) y unidades sin arranque en negro (NBS por sus siglas en inglés). Las unidades BS son limitadas y pueden arrancar autónomamente sin necesidad de una fuente externa de alimentación; por otro lado, las unidades NBS requieren tensión a través de una fuente externa para ponerse en marcha. Adicionalmente, se debe tener en cuenta que los recursos de generación poseen inflexibilidades debido a sus características técnicas como tiempos mínimos de arranque y parada, cambios de potencia permitidos entre periodos y mínimos técnicos, entre otros (Shen et al., 2018). Debido al número limitado generadores BS y a las diferentes restricciones para el arranque de unidades de generación, el problema radica en determinar la máxima disponibilidad de generación encontrando la secuencia óptima de arranque de todas las unidades.

Las investigaciones relacionadas con la secuencia de arranque óptimo pueden ser divididas en dos categorías: métodos de toma de decisiones y métodos de optimización múltiple. En (Wen et al., 2014) se desarrolla un modelo de toma de decisiones de dos etapas para determinar la secuencia de arranque de acuerdo a ciertas reglas o políticas establecidas. La primera etapa consiste en optimizar la generación disponible y la segunda en determinar la nueva reconfiguración topológica de la red. En (Gu y Zhong, 2012), la secuencia de arranque óptima es tratada como un proceso de restablecimiento de dos capas, denominadas unidades de restablecimiento de trabajo y de planta. En esta propuesta, se utiliza el método de optimización lexicográfica para solucionar el problema de optimización multi-objetivo resultante. En (Ketabi et al., 2019), se aborda el problema de restablecimiento de arranque de unidades de generación mediante optimización binivel. En este caso, el nivel superior determina la secuencia de arranque óptima de unidades NBS mediante el uso de algoritmos de optimización basados en enseñanza-aprendizaje y el nivel inferior determina el camino de transmisión óptimo con un mínimo número de movimientos de interruptores y máxima confiabilidad entre nodos utilizando teoría de grafos.

En (Golshani et al., 2018), se aborda el problema de restablecimiento considerando el efecto de la generación eólica. En este caso se propone un problema de adaptación robusto de dos etapas. En la primera etapa se determina la secuencia óptima de arranque de generadores y el restablecimiento de la red de transmisión; en la segunda etapa se optimiza la secuencia de cargas a reconectar. Un abordaje similar es propuesto en (Sun et al., 2018) en el que la primera etapa es dividida en dos sub-problemas de optimización: la secuencia de arranque de generadores y la determinación de una red troncal de transmisión factible para la restauración; mientras en la segunda etapa se determina la secuencia óptima de cargas a restablecer. En (Li et al., 2017) se propone un método de seccionamiento de red en paralelo con el objetivo de minimizar el tiempo de restablecimiento. Inicialmente, se establece una red troncal mediante un algoritmo de árbol de expansión mínimo, luego se divide la red en subsistemas dependiendo del número de generadores BS disponibles. Finalmente, con base en la red troncal y los subsistemas se identifica el esquema de reconexión óptimo de la red.

Desde el punto de vista de programación matemática el problema de restablecimiento es no lineal entero mixto y presenta el fenómeno de explosión combinatoria. Por esta razón diferentes técnicas metaheurísticas también han sido empleadas para la solución de este problema. Estas incluyen algoritmos evolutivos (Talib et al., 2017), redes neuronales artificiales (Bretas y Phadke, 2003), lógica difusa (Hsiao y Chien, 2000), optimización basada en el comportamiento de las luciérnagas (El-Zonkoly, 2015) y redes de Petri (Wu et al., 2000). En (Liu et al., 2016) se presenta una revisión de técnicas de optimización aplicadas al problema de restablecimiento. La principal debilidad de las técnicas metaheurísticas es que necesitan entrenamiento o ajuste de varios parámetros, lo cual limita aplicaciones en tiempo real. En este trabajo se presenta un modelo de programación lineal entero mixto para abordar el problema de restablecimiento en un SEP que garantiza la obtención de óptimos globales en tiempos de solución viables para aplicaciones en tiempo real. En este caso, se aborda la primera etapa del problema de restablecimiento que consiste en determinar la secuencia óptima de arranque de generadores BS y NBS teniendo en cuenta las restricciones de generación; por otro 
lado, la reconexión de las cargas se hace siguiendo el orden de prioridad definido por el operador de red. Se realizan pruebas en dos sistemas que evidencian la robustez del modelo y su aplicabilidad en sistemas reales.

\section{MODELO MATEMÁTICO PARA LA RESTAURACIÓN DEL SISTEMA}

En el desarrollo de este trabajo se han tomado como guía las funciones objetivo propuestas en (Sun et al., 2011) y (El-Zonkoly, 2015), las cuales plantean un objetivo directo y uno indirecto. El objetivo directo consiste en maximizar la capacidad de generación del sistema $E_{\text {gen }}$ sys; mientras el objetivo indirecto consiste en atender la energía no suministrada $E N S_{\text {sys. }}$

\section{Función Objetivo}

La Egen $_{\text {sys }}$ se define como la capacidad total del sistema, menos los requerimientos de arranque de los generadores; como se muestra en la ecuación (1).

$$
\text { Egen }_{\text {sys }}=\sum_{i=1}^{N} E_{\text {igen }}-\sum_{j=1}^{M} E_{j s t a r t}
$$

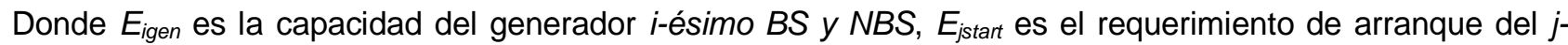
ésimo generador NBS, Nes el número total de generadores y $M$ es el número de generadores NBS. La Figura 1 caracteriza la capacidad de los generadores BS y NBS; y los requerimientos de arranque de un generador NBS.

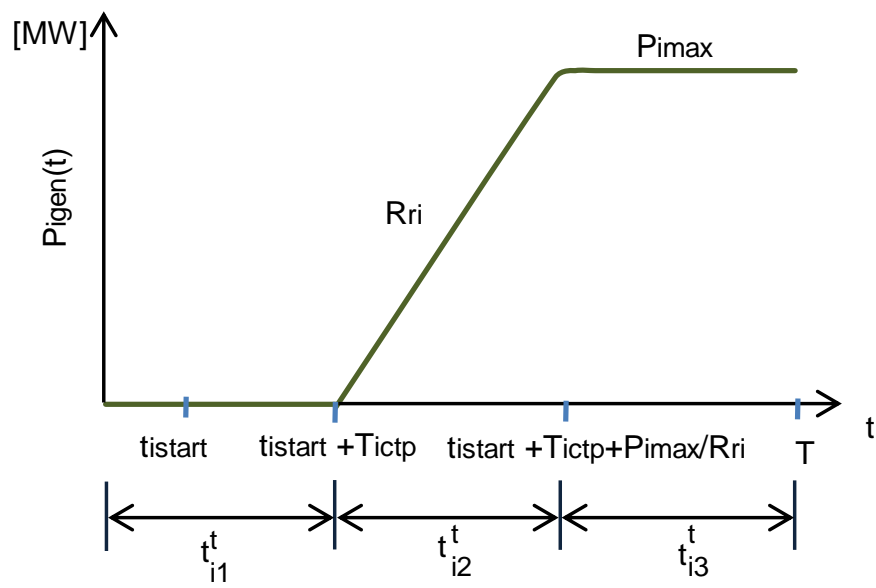

(a)

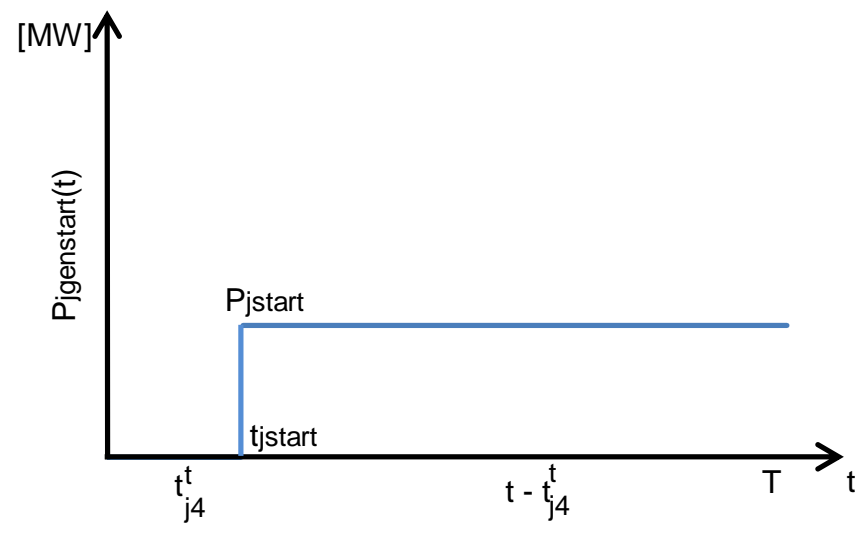

(b)

Fig.1: Caracterización de generadores convencionales de un SEP. (a) Curva de capacidad de generadores BS y NBS. (b) Curva de arranque de un generador NBS

El área bajo la curva de la capacidad de generación y el eje horizontal representan la capacidad total en MW del sistema sobre un período $T$ de restablecimiento. En la Figura 1(a), para el i-ésimo generador se tiene: 
$P_{\text {imax }}$ es la máxima potencia que puede entregar un generador, $t_{\text {istart }}$ es el tiempo de arranque, $T_{\text {ictp }}$ es el tiempo desde el arranque hasta la rampa de subida, $R_{r i}$ es la rampa de subida y $T$ es el período de restablecimiento dado. La ecuación (2) representa el área bajo la curva de la capacidad de generación.

$$
E_{\text {igen }}=\frac{1}{2} P_{\text {imax }} \frac{P_{\text {imax }}}{R_{r i}}+P_{\text {imax }}\left[T-\left(t_{\text {istart }}+T_{\text {ictp }}+\frac{P_{\text {imax }}}{R_{r i}}\right)\right]
$$

Por otra parte, el área bajo la curva del requerimiento de arranque y el eje horizontal representan la energía de arranque que necesita un generador NBS, la cual es proporcionada desde un generador BS. En la Figura 1(b), para el j-ésimo generador NBS se tiene: $P_{j s t a r t}$ es la potencia mínima de arranque, $t_{j s t a r t}$ es el tiempo de arranque y $T$ es el período de restablecimiento dado. La ecuación (3) representa el área bajo la curva del arranque de un generador NBS.

$$
E_{\text {jstart }}=P_{\text {jstart }}\left(T-t_{\text {jstart }}\right)
$$

Usando las ecuaciones (2) y (3), la energía disponible en el sistema $E_{g e n} n_{s y s}$ se puede representar como se muestra en la ecuación (4).

$$
\begin{gathered}
\text { Egen }_{\text {sys }}=\left\{\sum_{i=1}^{N}\left[\frac{\left(P_{\text {imax }}\right)^{2}}{2 R_{r i}}+P_{\text {imax }}\left(T-T_{\text {ictp }}-\frac{P_{\text {imax }}}{R_{r i}}\right)\right]-\sum_{j=1}^{M} P_{j s t a r t} T\right\} \\
-\left(\sum_{i=1}^{N} P_{\text {imax }} t_{\text {istart }}-\sum_{j=1}^{M} P_{j s t a r t} t_{\text {jstart }}\right)
\end{gathered}
$$

La ecuación (4) muestra que la capacidad de generación consta de dos partes. La primera componente que se encuentra en corchetes es constante. La segunda componente está en función de la variable de decisión $t_{\text {start. }}$ Se asumirá que para los generadores BS, el arranque se realizará al inicio del restablecimiento en $t=0$. Con esto, la primera suma de la segunda componente en la ecuación (4) se puede reducir de $\mathrm{Na} \mathrm{M}$. Por lo tanto, la capacidad de generación del sistema se puede simplificar como se muestra en la ecuación (5).

$$
\text { Egen }_{\text {sys }}=\sum_{j=1}^{N}\left(P_{j \max }-P_{\text {jstart }}\right) t_{\text {jstart }}
$$

Por otra parte, la $E N S_{s y s}$ se define como el área bajo la curva de la demanda desatendida total del sistema $P_{L s y s}$ en MW menos la cl-ésima demanda restablecida $P L_{c l}$ en un período de tiempo $T$. La Figura 2 esquematiza el concepto de energía no suministrada aplicado en esta investigación.

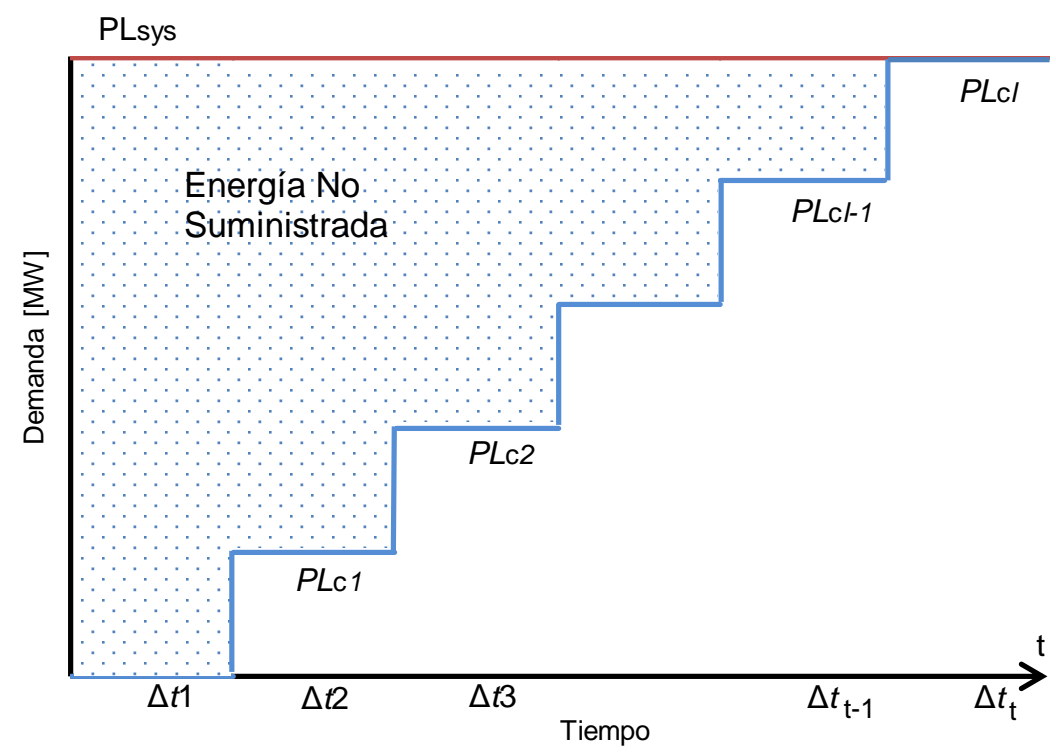

Fig.2: Energía no suministrada (ENS) del sistema

Si se divide el período de tiempo $T$ en $\Delta t$ períodos de la misma longitud; la $E N S_{\text {sys }}$ se calcula como el área bajo la curva de la sumatoria de todos los rectángulos de lado $P_{L s y s}-P_{L c l}$ por lado $\Delta t_{t}$, tal como se establece en la ecuación (6). 


$$
E N S_{s y s}=\sum_{t=1}^{T} \sum_{l=1}^{L}\left(P_{L s y s}-P_{L c l}\right) \Delta t_{t}
$$

Definidas las funciones de capacidad de generación y energía no suministrada, la función objetivo del problema de optimización puede definirse en dos componentes como se muestra en la ecuación (7). La primera componente indica que para maximizar la capacidad de generación del sistema en un restablecimiento, basta con minimizar los tiempos de arranque de los generadores. La segunda buscará minimizar la demanda no atendida del sistema.

$$
\text { minimizar } \sum_{i=1}^{N}\left(P_{\text {jmax }}-P_{\text {jstart }}\right) t_{\text {jstart }}+\sum_{t=1}^{T} \sum_{c l=1}^{L}\left(P_{L s y s}-P_{L c l}\right) \Delta t_{t}
$$

\section{Restricciones del problema de optimización}

Tiempos críticos máximos y mínimos: Esta restricción se establece para los generadores NBS, debido a las características físicas y termodinámicas de las unidades de generación. Si una unidad NBS no arranca dentro de un intervalo de tiempo máximo crítico $T_{c m a x}$, la unidad no estará disponible hasta después de un tiempo considerable de retraso. Por otro lado, una unidad NBS con una restricción de intervalo de tiempo crítico mínimo $T_{c m i n}$, no se puede restablecer hasta que este tiempo expire. Con estas consideraciones, los generadores deben satisfacer las ecuaciones (8) y (9).

$$
\begin{aligned}
& t_{\text {jstart }} \geq T_{j \text { cmin }} \\
& t_{\text {jstart }} \leq T_{j \text { cmax }}
\end{aligned}
$$

Requerimientos de potencia de Arranque: Los generadores NBS solo pueden ser arrancados cuando el sistema puede suministrar la suficiente potencia de arranque, para cada período de tiempo. Esta restricción se representa mediante la ecuación (10).

$$
\sum_{i=1}^{N} P_{i g e n}(t)-\sum_{j=1}^{M} P_{j s t a r t}(t) \geq 0
$$

La ecuación (10) lleva a un problema de optimización combinatorio no lineal. Sin embargo, mediante una adecuada transformación de las formulaciones de la función de capacidad de generación $P_{\text {igen }}(t)$ y la función de requerimiento de arranque para unidades NBS convencionales $P_{\text {istart }}(t)$; se puede lograr una formulación de un problema de programación lineal entero mixto. La curva de capacidad de generación representada en la Figura 1(a), se puede modelar como una función a tramos. Los puntos $\left(T_{i c t p}+t_{\text {istart, }} 0\right)$ y $\left(T_{\text {ictp }}+t_{\text {istart }}+P_{\text {imax }} / R_{r i}\right.$, $P_{\text {imax }}$ ), dividen la curva en tres segmentos $t^{t_{11}}, t_{i 2}{ }^{t}$ y $t_{i 3}{ }^{t}$. Esta representación modela el comportamiento del arranque de generación desde el apagón, hasta que alcanza la potencia máxima disponible por característica técnica.

Por otra parte, la curva de arranque de generación de la Figura 1(b); se modela como una función a tramos discontinua de dos segmentos. El primer segmento $\mathrm{t}_{\mathrm{j}}^{\mathrm{t}} \mathrm{k}$, representa el tiempo desde el apagón hasta un instante antes que arranque el generador. El segundo segmento $t-t^{t} j 4$, representa el tiempo desde el momento en que la unidad arrancó hasta el tiempo total de restablecimiento $T$. Adicionalmente, se utilizan las variables binarias $w_{i 1}^{t}, w_{i 2}^{t} y w_{i 3}^{t}$ para restringir y definir los respectivos rangos de tiempos donde se encuentre el proceso de optimización. Con esto, las curvas de capacidad de generación y de arranque se modelan matemáticamente de acuerdo a lo establecido en las ecuaciones (11) a la (23).

$$
\begin{aligned}
& P_{i g e n}(t)=R_{r i} t_{i 2}^{t} \\
& t=t_{i 1}^{t}+t_{i 2}^{t}+t_{i 3}^{t} \\
& w_{k 1}^{t} T_{k c t p} \leq t_{k 1}^{t} \leq T_{k c t p} \\
& w_{j 1}^{t}\left(t_{j s t a r t}+T_{j c t p}\right) \leq t_{j 1}^{t} \leq t_{j s t a r t}+T_{j c t p}
\end{aligned}
$$




$$
\begin{aligned}
& w_{i 2}^{t} \frac{P_{\text {imax }}}{R_{r i}} \leq t_{i 2}^{t} \leq w_{i 1}^{t} \frac{P_{\text {imax }}}{R_{r i}} \\
& 0 \leq t_{k 3}^{t} \leq w_{k 2}^{t}\left(T-T_{k c t p}-\frac{P_{\text {kmax }}}{R_{r k}}\right) \\
& 0 \leq t_{j 3}^{t} \leq w_{j 2}^{t}\left(T-t_{j s t a r t}-T_{j c t p}-\frac{P_{\text {jmax }}}{R_{r j}}\right) \\
& w_{i 2}^{t} \leq w_{i 1}^{t} \\
& P_{j s t a r t}(t)=w_{j 3}^{t} P_{j s t a r t} \\
& w_{j 3}^{t}\left(t_{j s t a r t}-1\right) \leq t_{j 4}^{t} \leq t_{j s t a r t}-1 \\
& w_{j 3}^{t} \leq t-t_{j 4}^{t} \leq w_{j 3}^{t}\left(T-t_{j s t a r t}+1\right) \\
& w_{k}^{t}, w_{j}^{t}, w_{i}^{t} \in\{0,1\} \\
& t_{i 1}^{t}, t_{i 2}^{t}, t_{i 3}^{t}, t_{j 4}^{t} \in(0,1,2, \ldots, T)
\end{aligned}
$$

Finalmente, con el desarrollo realizado en las ecuaciones (11) a (23), la restricción de requerimiento de arranque de generación de la ecuación (10) puede reescribirse como se muestra en la ecuación (24).

$$
\sum_{i=1}^{N} R_{r i} t_{i 2}^{t}-\sum_{j=1}^{M} w_{j 3}^{t} P_{j s t a r t} \geq 0, \quad t=1,2, \ldots, T
$$

Restablecimiento de la demanda: Las curvas de atención de demanda en función del tiempo PLcarga(t), se modelan como funciones escalón. La demanda será atendida en el momento que se tenga la suficiente potencia en el sistema. La Figura 3 muestra que la curva de restablecimiento de demanda se modela como una función a tramos de dos segmentos. El primer segmento $\mathrm{t}^{\mathrm{t}} 15$, representa el tiempo desde el apagón hasta un instante de tiempo antes de normalizar la demanda. El segundo segmento $\mathrm{t}^{t}-1 \mathrm{t}^{\mathrm{t}} \mathrm{t}$, representa el tiempo desde que se normaliza la demanda $P_{\text {Lcl }}$ hasta el tiempo total de restablecimiento $T$. Las ecuaciones (25) a (29) modelan este comportamiento.

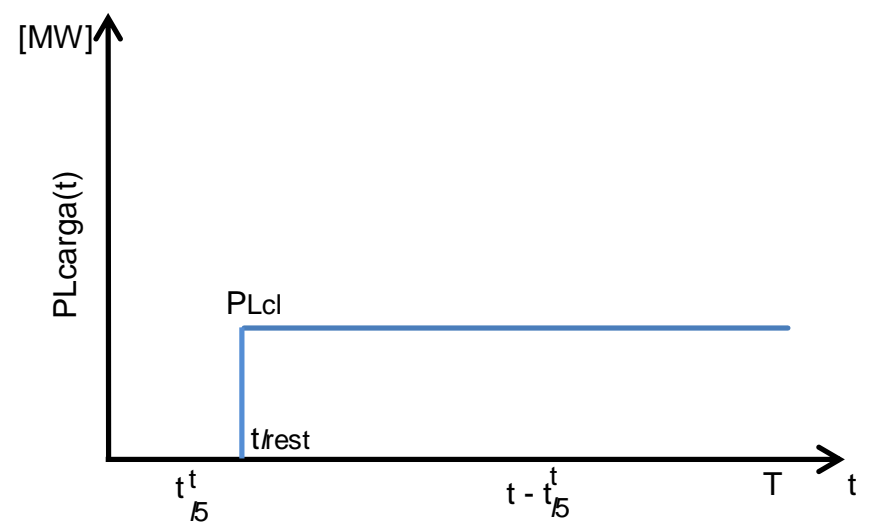

Fig. 3: Función escalón unitario que caracteriza el restablecimiento de la demanda

$$
\begin{aligned}
& P_{\text {Lcarga }}(t)=w_{l 4}^{t} P_{L c l} \\
& w_{l 4}^{t}\left(t_{\text {lrest }}-1\right) \leq t_{l 5}^{t} \leq t_{\text {lrest }}-1 \\
& w_{l 4}^{t} \leq t^{t}-t_{l 5}^{t} \leq w_{l 4}^{t}\left(T-t_{\text {lrest }}+1\right) \\
& w_{l 4}^{t} \in\{0,1\} \\
& t_{l 5}^{t} \in(0,1,2, \ldots, T)
\end{aligned}
$$


Prioridad de la demanda: El restablecimiento de la demanda del sistema, debe realizarse en un orden prioritario, con el objetivo de hacerlo más efectivo y maximizar el beneficio social. La ecuación (30) modela la atención de cargas prioritarias o subestaciones que se requiere energizar primero como por ejemplo centros de control, acueductos u hospitales.

$$
t_{\text {lrest }} \leq t_{(l+1) \text { rest }}
$$

Balance carga-generación: La ecuación (31) modela el hecho que la demanda será restablecida siempre y cuando exista en el sistema la potencia suficiente para normalizarla.

$$
\sum_{i=1}^{N} R_{r i} t_{i 2}^{t}-\sum_{j=1}^{M} w_{j 3}^{t} P_{j s t a r t} \geq \sum_{l=1}^{L} w_{l 4}^{t} P_{L c l}, \quad t=1,2, \ldots, T
$$

Linealización de ecuaciones: Las restricciones desarrolladas en las ecuaciones (14), (17), (20), (21), (26) y (27) son de tipo no lineal. Para linealizar estas ecuaciones, se introducirá al modelo una nueva variable continua $y^{t}$ in como el producto de una variable entera por una binaria $\left(w^{t}{ }_{i h} \times t_{i}\right)$. El cambio de variable debe cumplir las restricciones mostradas en las ecuaciones (32) a (37).

$$
\begin{aligned}
& y_{i h}^{t}=w_{i h}^{t} \cdot t_{i} \\
& y_{i h}^{t} \geq 0 \\
& y_{i h}^{t} \leq M w_{i h}^{t} \\
& -t_{i}+y_{i h}^{t} \leq 0 \\
& t_{i}-y_{i h}^{t}+M w_{i h}^{t} \leq M \\
& t_{i} \leq M
\end{aligned}
$$

\section{PRUEBAS Y RESULTADOS}

Para validar el modelo propuesto se utilizan dos sistema de prueba, un sistema didáctico de 4 generadores y el sistema IEEE-39 RTS. En todas las pruebas se utilizó un computador con procesador Intel(R) core(TM) i54200U @ 1.6 GHz 2.3 GHz, 6,00 GB de memoria RAM y sistema operativo de 64 bits.

\section{Resultados con un sistema de 4 generadores}

Inicialmente se realizan pruebas con un sistema didáctico de 4 generadores, en el que solo uno es BS. Las características de generación y carga de este sistema se indican en las Tablas 1 y 2, respectivamente.

Tabla 1: Parámetros de los generadores del sistema de prueba

\begin{tabular}{|c|c|c|c|c|c|c|c|c|}
\hline GEN & Tipo & $T_{c t p}$ & $T_{\text {cmin }}$ & $T_{\text {cmax }}$ & $R_{r}[\mathrm{MW} / \mathrm{u} . \mathrm{t}]$ & $P_{\text {start }}[\mathrm{MW}]$ & $P_{\max }[\mathrm{MW}]$ & $\mathrm{H}[\mathrm{s}]$ \\
\hline 1 & NBS & 2 & $\mathrm{~N} / \mathrm{A}$ & 5 & 2 & 1 & 8 & 4 \\
\hline 2 & $\mathrm{NBS}$ & 1 & 5 & $\mathrm{~N} / \mathrm{A}$ & 4 & 1 & 12 & 5 \\
\hline 3 & $\mathrm{NBS}$ & 2 & $\mathrm{~N} / \mathrm{A}$ & 4 & 4 & 2 & 20 & 3 \\
\hline 4 & $\mathrm{BS}$ & 1 & $\mathrm{~N} / \mathrm{A}$ & $\mathrm{N} / \mathrm{A}$ & 1 & $\mathrm{~N} / \mathrm{A}$ & 3 & 1 \\
\hline
\end{tabular}

Tabla 2: Parámetros de la carga del sistema de prueba

\begin{tabular}{|c|c|c|}
\hline Demanda & Prioridad & $P l_{\text {carga }}[\mathrm{MW}]$ \\
\hline 1 & 2 & 10 \\
\hline 2 & 1 & 3 \\
\hline 3 & 3 & 6 \\
\hline 4 & 5 & 7 \\
\hline 5 & 4 & 5 \\
\hline
\end{tabular}

Los tiempos de arranque de los generadores NBS se muestran en la Tabla 3 (donde u.t significa unidad de tiempo). Al realizar la corrida del modelo de optimización se obtiene una función objetivo de $345 \mathrm{MW} / \mathrm{ut}$ en un tiempo de ejecución de 0,12 segundos en 79 iteraciones. A medida que va arrancando cada unidad de generación y de acuerdo a las características técnicas de las unidades va aumentando la generación disponible en el sistema como se ilustra en la Figura 4. 
Tabla 3: Tiempo de arranque de las unidades de generación

\begin{tabular}{|c|c|c|}
\hline GEN & Tipo & Tiempo de arranque [u.t] \\
\hline 1 & NBS & 2 \\
\hline 2 & NBS & 5 \\
\hline 3 & NBS & 4 \\
\hline 4 & BS & 0 \\
\hline
\end{tabular}

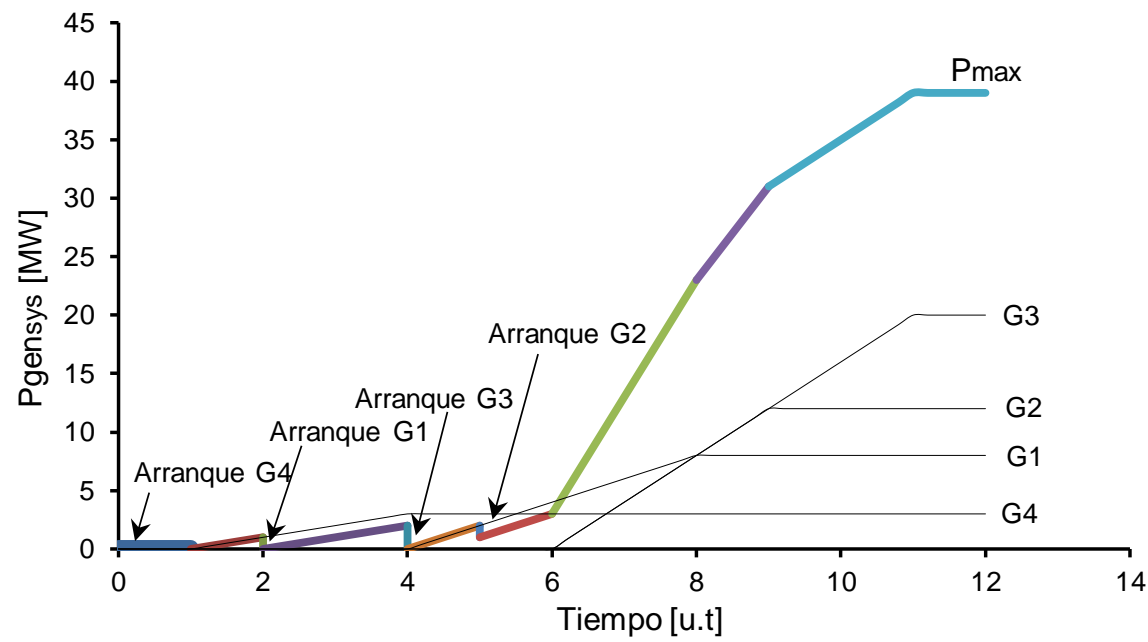

Fig. 4: Curva de capacidad del sistema de prueba en un restablecimiento

En la Tabla 4 se presenta el estado de cada generador para la solución óptima. En t=0 el generador BS G4 es arrancado. En t=1 ya se ha completado el tiempo $T_{\text {ictp }}$ de la unidad BS. Por lo tanto, inicia su rampa de arranque. $\mathrm{En} \mathrm{t}=4$, G4 ha aportado $1 \mathrm{MW}$ al sistema haciendo que se cumplan las condiciones para arrancar el generador G1. En $\mathrm{t}=4$ ya se ha completado el tiempo $T_{\text {ictp }}$ de la unidad NBS $\mathrm{G}$ y comienza su rampa de subida. Por otra parte, también se dan las condiciones para que arranque G3; por otro lado, G4 alcanza su máxima potencia ayudando al arranque de $\mathrm{G} 1$ y G3. En $\mathrm{t}=5$ se cumplen las condiciones para el arranque de $\mathrm{G} 2$. En $\mathrm{t}=6$ expiran los $T_{\text {ictp }}$ de G2 y G3, comenzando en este instante sus rampas de subida. Por otro lado, G1 continúa su rampa de ascenso. En $\mathrm{t}=8, \mathrm{G} 1$ alcanza su máxima capacidad de generación. Posteriormente en $\mathrm{t}=9$, $\mathrm{G} 2$ alcanza su máxima capacidad de generación y finalmente en $\mathrm{t}=11$, G3 alcanza su máxima capacidad de generación, maximizando la capacidad de generación del sistema. Se observa que en $\mathrm{t}=12$ el sistema de prueba tiene $39 \mathrm{MW}$ disponibles, lo que equivale a $43 \mathrm{MW}$ de la potencia máxima de todas las unidades, menos $4 \mathrm{MW}$ de los requerimientos de arranque de las unidades NBS.

Tabla 4: Solución óptima para los estados de los generadores

\begin{tabular}{|c|c|c|c|c|c|}
\hline Tiempo & G1 & G2 & G3 & G4 & Capacidad (MW) \\
\hline $\mathrm{t}=0$ & 0 & 0 & 0 & 1 & 0 \\
\hline $\mathrm{t}=1$ & 0 & 0 & 0 & 1 & 0 \\
\hline $\mathrm{t}=2$ & 1 & 0 & 0 & 1 & 0 \\
\hline $\mathrm{t}=4$ & 1 & 0 & 1 & 1 & 0 \\
\hline $\mathrm{t}=6$ & 1 & 1 & 1 & 1 & 3 \\
\hline $\mathrm{t}=12$ & 1 & 1 & 1 & 1 & 39 \\
\hline
\end{tabular}

De acuerdo con la curva de capacidad agregada de generación del sistema, se empieza a normalizar la demanda siguiendo el orden de prioridad que se estableció previamente. En la Figura 5 se ilustran simultáneamente las gráficas de generación disponible en el sistema y la ENS. Se puede observar que a medida en que ingresan los generadores al sistema, se va restableciendo la demanda no atendida en el orden de prioridad establecido en la Tabla 5. 
Tabla 5: Tiempos de restablecimiento de la demanda

\begin{tabular}{|c|c|c|}
\hline Demanda & Valor & Tiempo de restablecimiento [u.t] \\
\hline 1 & 10 & 7 \\
\hline 2 & 3 & 5 \\
\hline 3 & 6 & 8 \\
\hline 4 & 7 & 9 \\
\hline 5 & 5 & 9 \\
\hline
\end{tabular}
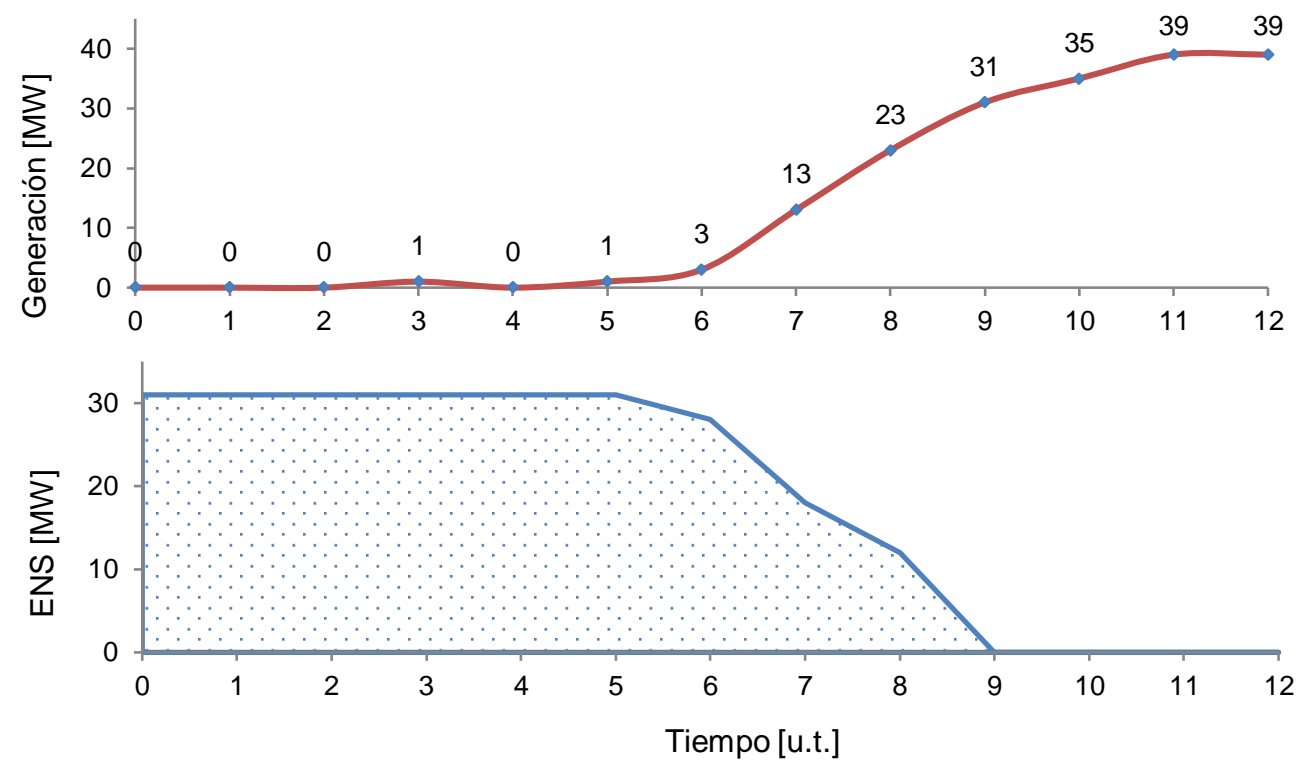

Fig. 5: Generación y ENS para el sistema de prueba de 4 generadores

En $\mathrm{t}=6$, se normaliza la demanda 2, la cual tiene una potencia de 3MW los cuales están disponibles en el sistema. $\mathrm{En}=7$, a medida que empiezan a arrancar los generadores NBS hay condiciones para normalizar la demanda 1 la cual tiene el orden 2 de prioridad. Existen $13 \mathrm{MW}$ en el sistema, utilizados para energizar los 3 de la demanda 2 más los 10 de la demanda 1 . En $\mathrm{t}=8$ el sistema ha sumado $10 \mathrm{MW}$ adicionales de capacidad de generación. En este punto, solamente se puede normalizar la demanda 3 que tiene prioridad 3 con un valor de 6 MW. Finalmente, en $\mathrm{t}=9$ se normalizan las demandas 4 y 5 . En este punto, se tienen las condiciones necesarias de generación para normalizar los $12 \mathrm{MW}$ restantes de carga; reduciendo a cero la ENS en el sistema.

\section{Resultados con el sistema IEEE- 39 RTS}

Este sistema cuenta con 10 generadores cuyas características se presentan en la Tabla 6 . En este caso, se tendrá un periodo de evaluación de 4 horas y media con una granularidad de 5 minutos, lo que equivale a 55 periodos de tiempo. En la Tabla 7 se presentan las cargas asociadas al sistema de prueba; se asume que el escenario es un apagón total.

Tabla 6: Parámetros de generadores del sistema IEEE-39 RTS

\begin{tabular}{|c|c|c|c|c|c|c|c|}
\hline GEN & Tipo & $\mathrm{T}_{\text {ctp }}[\mathrm{h}]$ & $\mathrm{T}_{\text {cmin }}[\mathrm{h}]$ & $\mathrm{T}_{\text {cmax }}[\mathrm{h}]$ & $\mathrm{R}_{\mathrm{r}}[\mathrm{MW} / \mathrm{h}]$ & $\mathrm{P}_{\text {start }}[\mathrm{MW}]$ & $\mathrm{P}_{\max }[\mathrm{MW}]$ \\
\hline 1 & NBS & $00: 35$ & $00: 40$ & $\mathrm{~N} / \mathrm{A}$ & 215 & 5,5 & 520 \\
\hline 2 & NBS & $00: 35$ & $\mathrm{~N} / \mathrm{A}$ & $\mathrm{N} / \mathrm{A}$ & 246 & 8 & 650 \\
\hline 3 & $\mathrm{NBS}$ & $00: 35$ & $\mathrm{~N} / \mathrm{A}$ & $02: 00$ & 236 & 7 & 632 \\
\hline 4 & $\mathrm{NBS}$ & $00: 35$ & $01: 10$ & $\mathrm{~N} / \mathrm{A}$ & 198 & 5 & 508 \\
\hline 5 & $\mathrm{NBS}$ & $00: 35$ & $\mathrm{~N} / \mathrm{A}$ & $01: 00$ & 244 & 8 & 650 \\
\hline 6 & $\mathrm{NBS}$ & $00: 35$ & $\mathrm{~N} / \mathrm{A}$ & $\mathrm{N} / \mathrm{A}$ & 214 & 6 & 560 \\
\hline 7 & $\mathrm{NBS}$ & $00: 35$ & $\mathrm{~N} / \mathrm{A}$ & $\mathrm{N} / \mathrm{A}$ & 210 & 6 & 540 \\
\hline 8 & $\mathrm{NBS}$ & $00: 35$ & $\mathrm{~N} / \mathrm{A}$ & $\mathrm{N} / \mathrm{A}$ & 346 & 13,2 & 830 \\
\hline 9 & $\mathrm{NBS}$ & $00: 35$ & $\mathrm{~N} / \mathrm{A}$ & $\mathrm{N} / \mathrm{A}$ & 384 & 15 & 1000 \\
\hline 10 & $\mathrm{BS}$ & $00: 15$ & $\mathrm{~N} / \mathrm{A}$ & $\mathrm{N} / \mathrm{A}$ & 162 & 0 & 250 \\
\hline
\end{tabular}


Tabla 7: Datos de demanda sistema IEEE-39 RTS

\begin{tabular}{|c|c|c|c|c|c|c|c|c|}
\hline Nodo & Prioridad & $P l_{\text {carga }}[\mathrm{MW}]$ & Nodo & Prioridad & $P_{\text {carga }}[\mathrm{MW}]$ & Nodo & Prioridad & $P l_{\text {carga }}[\mathrm{MW}]$ \\
\hline 3 & 1 & 322 & 18 & 8 & 281 & 27 & 15 & 158 \\
\hline 4 & 2 & 500 & 20 & 9 & 628 & 28 & 16 & 206 \\
\hline 7 & 3 & 234 & 21 & 10 & 274 & 29 & 17 & 284 \\
\hline 8 & 4 & 522 & 23 & 11 & 247 & 31 & 18 & 9 \\
\hline 12 & 5 & 8 & 24 & 12 & 309 & 39 & 19 & 1104 \\
\hline 15 & 6 & 320 & 25 & 13 & 224 & & & \\
\hline 16 & 7 & 328 & 26 & 14 & 139 & & & \\
\hline
\end{tabular}

Después de realizar la corrida del modelo de optimización, se obtuvo una función objetivo de 227278,4 [MW/Hr] en un tiempo de 27,16 [s]. La Figura 6 muestra los tiempos de arranque obtenidos para los generadores NBS parametrizados en la Tabla 6. El arranque de generadores se realiza entre las 00:20 Hrs y 01:15 Hrs después de ocurrido el apagón; donde se cumplen las condiciones de restricciones de tiempos críticos máximos y mínimos, y de potencia de arranque mínima de generación.

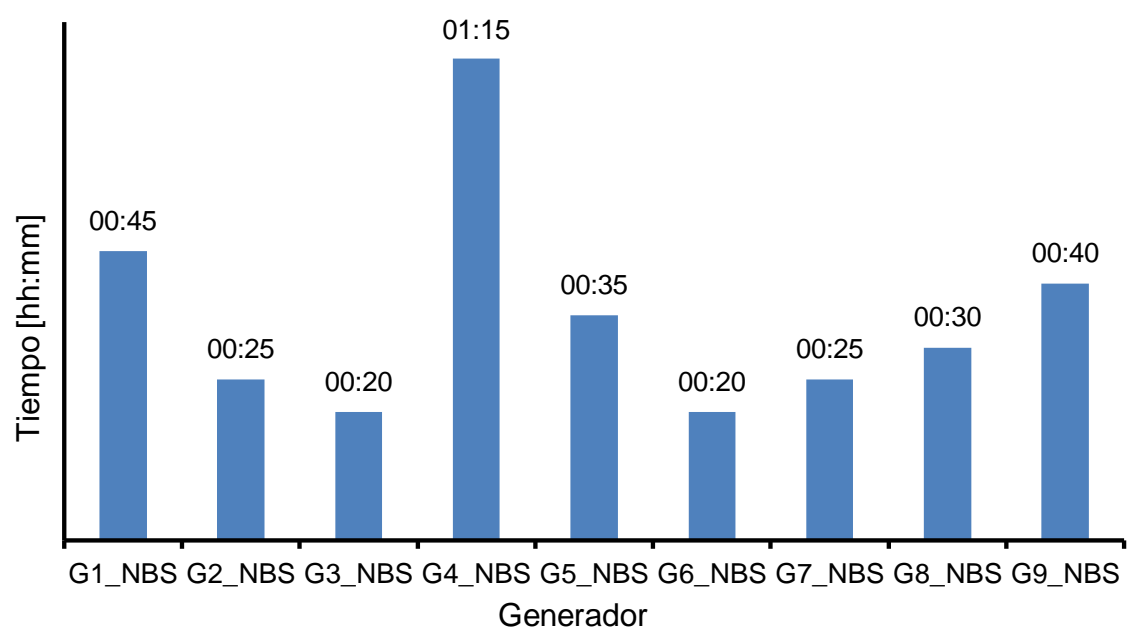

Fig. 6: Tiempo de arranque de generadores sistema IEEE-39 RTS

Por ejemplo, el generador 1 arranca a las 00:45 Hrs donde su tiempo mínimo de arranque son 00:40 Hrs y el generador 4 arranca a la 01:15 Hrs donde su tiempo mínimo de arranque son 01:10 Hrs. Por otra parte, se cumplen los tiempos máximos de arranque, donde los generadores 3 y 5 arrancan antes de las 02:00 y 01:00 horas respectivamente (ver Tabla 6). En la Figura 6, se muestran los resultados obtenidos de la generación acumulada del sistema después del apagón y la ENS que se presenta durante el evento. Se observa que a medida que se van sincronizando generadores al sistema, se va restableciendo la demanda de acuerdo al orden de prioridad establecido en la Tabla 7.

Los generadores empiezan su proceso de arranque y después que cumplen el tiempo Tctp le aportan generación al sistema. A la 01:10 Hrs se restablecen la primera carga de $322 \mathrm{MW}$ que tiene la primera prioridad de energización. En ese instante, la generación acumulada del sistema es de 430,2 MW por lo que se cumple la condición de balance carga-generación del sistema. Posteriormente, a la 01:35 Hrs se normaliza la demanda con prioridad 2 de $500 \mathrm{MW}$ donde se encuentran sincronizados en el sistema 976,3 MW. Este proceso se realiza hasta cuando se normalizan todas las demandas del sistema a las 4:15 Hrs de iniciado el proceso de restablecimiento. En la Figura 7 se ilustra la generación y ENS del sistema durante el proceso de restablecimiento.

\section{Comparación con otros métodos}

A continuación se presenta una comparación del modelo de optimización desarrollado en este artículo frente a otras metodologías presentadas en la literatura. En la Tabla 8 se presenta, para diferentes técnicas de optimización, el tiempo computacional y si fue alcanzado o no el óptimo global en el proceso de restablecimiento para el sistema de prueba IEEE-39 RST. Se puede observar que la metodología desarrollada en el presente artículo, proporciona una solución óptima global con un tiempo computacional satisfactorio. 


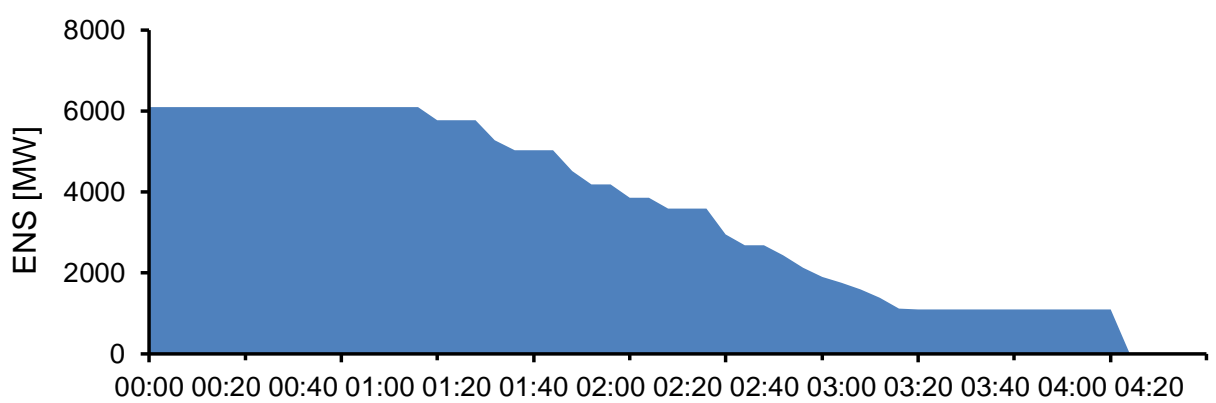

Tiempo [hh:mm]

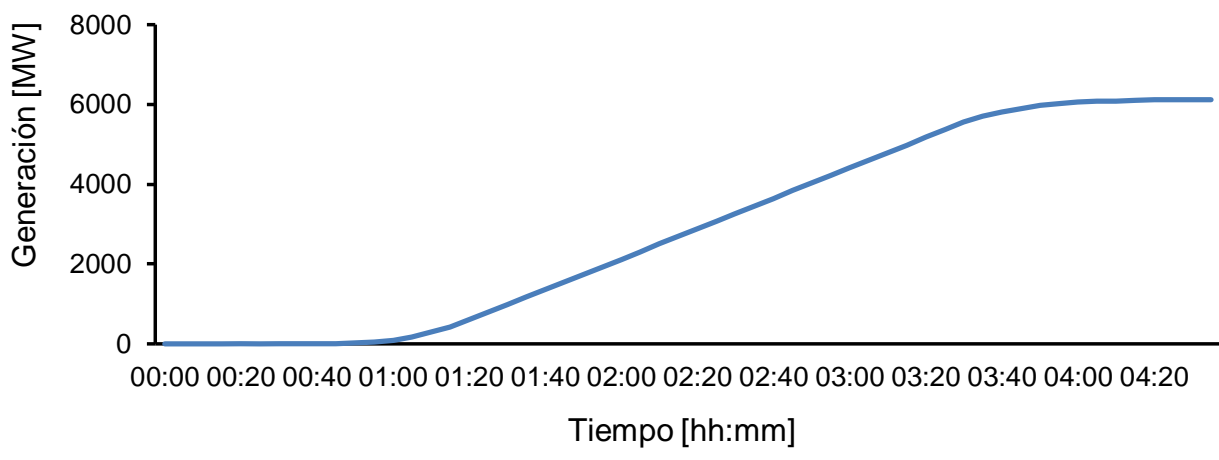

Fig. 7: Generación y ENS para el sistema IEEE-39 RTS

Tabla 8: Comparación con otros métodos reportados en la literatura técnica

\begin{tabular}{|l|l|c|c|}
\hline \multicolumn{1}{|c|}{ Referencia } & \multicolumn{1}{|c|}{ Algoritmo } & Tiempo de cómputo & ¿Óptimo global? \\
\hline (El-Zonkoly, 2015) & Enumeración & $01: 53: 00$ & Sí \\
\hline (Liu y Leader, 2009) & Algoritmo de dos pasos & $00: 04: 00$ & No \\
\hline Metodología propuesta & Programación lineal entera mixta & $00: 00: 28$ & Sí \\
\hline
\end{tabular}

Los resultados obtenidos por las técnicas presentadas fueron realizadas mediante diferentes computadores. El algoritmo de numeración fue procesado en un computador Core i3 @ $2.53 \mathrm{GHz}$. El algoritmo de dos pasos no referencia las características del computador de prueba. La metodología propuesta mediante MILP se realizó en un computador con procesador Intel(R) core(TM) i5-4200U @ $1.6 \mathrm{GHz} 2.3 \mathrm{GHz}$.

\section{CONCLUSIONES}

En este artículo se presentó un modelo de programación lineal entera mixta formulado mediante funciones lineales a tramos para resolver el problema de restablecimiento de sistemas de potencia. El modelo propuesto tiene en cuenta diferentes características técnicas de las unidades de generación y permite encontrar la secuencia óptima de arranque de los generadores de un sistema de potencia tras un apagón. La función objetivo minimiza simultáneamente los tiempos de arranque de las unidades de generación y la energía no suministrada al sistema. La formulación del problema utiliza un modelamiento matemático riguroso que tiene en cuenta de forma simultánea los tiempos de arranque críticos de las unidades, los requerimientos de potencia de arranque y el balance carga-generación del sistema.

Los resultados numéricos obtenidos en dos sistemas de prueba evidencian la efectividad y robustez del modelo propuesto. En particular, los resultados obtenidos con el sistema de prueba IEEE-39 RTS permiten concluir que el modelo puede ser implementado en aplicaciones reales. Esta conclusión se basa en dos características del modelo. La primera consiste en que la solución obtenida es un óptimo global (esto se da como consecuencia de haber planteado un modelo de programación lineal entera mixta). La segunda característica que facilita aplicaciones reales es la rapidez con que se obtiene la solución (secuencia de arranque de generadores). La comparación con otros métodos evidenció que el modelo tarda menos de un minuto en encontrar la solución para el sistema IEEE-39 RTS en un computador con especificaciones modestas, lo cual le da ventaja sobre otros métodos que tardan mucho más tiempo para encontrar la secuencia de arranque que permite el restablecimiento. 


\section{REFERENCIAS}

Bretas A.S. y Phadke, A.G., Artificial Neural Networks in Power System Restoration, https://doi.org/10.1109/TPWRD.2003.817500, IEEE Trans. Power Deliv., 18(4), 1181-1186, (2003).

El-Zonkoly, A., Integration of Wind Power for Optimal Power Black-Start Restoration, https://doi.org/10.3906/elk-1402-267, Turk. J. Elec. Eng. and Comp. Sci., 23(1), 1853-1866 (2015)

Golshani, A, Sun W., y otros cuatro autores, Coordination of Wind Farm and Pumped-Storage Hydro for a Pumped-Storage Hydro for a Self-Healing Power Grid, https://doi.org/10.1109/TSTE.2018.2819133, IEEE Trans. Sust. Energy, 9(4) 19101920 (2018)

Gu, X., y Zhong, H. Optimisation of Network Reconfiguration Based on a Two-Layer Unit-Restarting Framework for Power System Restoration, https://doi.org 10.1049/iet-gtd.2011.0591/, IET Gener. Transm. Distrib., 6(7) 693-700 (2012)

Hsiao, Y. y Chien, C., Enhancement of Restoration Service in Distribution Systems Using a Combination Fuzzy - GA Method, https://doi.org/10.1049/10.1109/59.898118 IEEE Trans. Power Syst, 15(4), 1394-1400 (2000)

Ketabi, A., Karimizadeh, A., y Shahidehpour, M., Optimal Generation Units Start-up Sequence During Restoration of Power System Considering Network Reliability Using Bi-Level Optimization https://doi.org/10.1016/j.ijepes.2018.07.045, Int. J. Electr. Power Energy Syst., 104(1), 772-783 (2019)

Li, C., He, J, Zhang, P., y Xu., Y., A Novel Sectionalizing Method for Power System Parallel Restoration Based on Minimum Spanning Tree, https://doi.org/10.3390/en10070948, Energies 10(7), 948-969 (2017)

Liu C.C. y Leader, P., Development and Evaluation of System Restoration Strategies from a Blackout Development and Evaluation of System, PSERC Research Project Report S-30. Iowa State University (2009)

Liu, Y., Fran, R., y Terzija, V., Power System Restoration: a Literature Review from 2006 to 2016 , https://doi.org/10.1007/s40565-016-0219-2, Journal of Modern Power Systems and Clean Energy 4(3), 332-341 (2016)

Quintero C.M., López-Lezama, J.M y Muñoz-Galeano, N., Técnicas para Aumentar la Seguridad de la Operación en Condiciones de Red Degradada a Través de Modificaciones Topológias en Sistemas de Potencia, http://dx.doi.org/10.4067/S0718-07642019000600363, Rev Inf. Tecnol., 30(6), 363-376 (2019)

Saldarriaga-zuluaga, S.D., López-Lezama, J.M y Muñoz-Galeano, N., Planeamiento Integrado de la Expansión en Generacion y Transmision Consideradon Restricciones de Seguridad, http://dx.doi.org/10.4067/S071807642018000300167, Rev Inf. Tecnol., 29(3), 167-176 (2018)

Shen, C., Kaufman, P., Hachmann, C., and Braun M., Three-stage Power System Restoration Methodology Considering Renewable Energies, https://doi.org/10.1016/j.ijepes.2017.07.007, Int. J. Electr. Power Energy Syst., 94, 287-299 (2018)

Sun L., Lin, Z. y otros cuatro autores, Optimal Skeleton-Network Restoration considering Generator Start-up Sequence and Load Pickup, https://doi.org/10.1109/TSG.2018.2820012, IEEE Trans Smart Grid 10(3), 3174-3185 (2018)

Sun, W., Liu, C.C., and Zhang, L., Optimal Generator Start-up Strategy for Bulk Power System Restoration, https://doi.org/10.1109/TPWRS.2010.2089646, Trans. Power Syst., 26(3), 1357-1366 (2011)

Talib D.N.A, Mokhlish, H., Talip MSA y Naidu, K, Parallel Power System Restoration Planning using Heuristic Initialization and Discrete Evolutionary Programming, https://doi.org/10.1007/s40565-017-0320-1, J Modern Power Syst. Clean Energy 5, 991-1003 (2017)

Wen, F., Ledwich, G. y otros tres autores, Two-stage Power Network Reconfiguration Strategy considering Node Importance and Restored Generation Capacity, https://doi.org/10.1049/iet-gtd.2013.0065, IET Gener. Transm. Distrib., 8(1), 91-103 (2014)

Wu, J.S., Liu, C.H. Liou, K.L. y Chu, R.F., A Petri Net Algorithm for Scheduling of Generic Restoration Actions, https://doi.org/10.1109/59.574925 IEEE Trans. Power Syst., 12(1), 538-545 (2000) 